
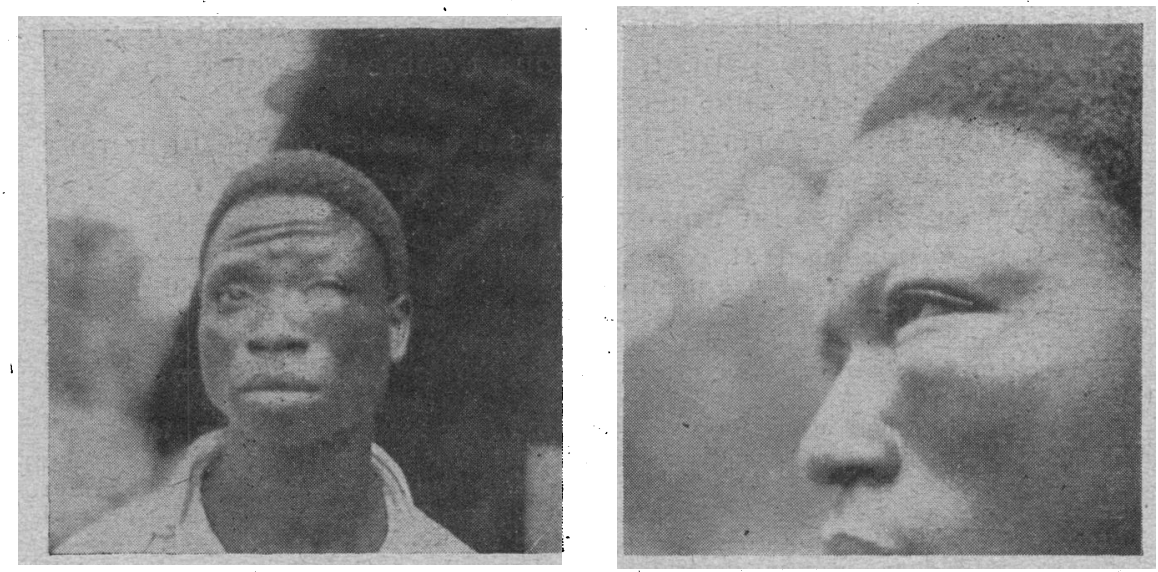

Cases of mucocele in Nigerians have been seen by the writer in connection with ethmoidal cells and the frontal sinus. As no case of this type has been seen, it may prove of interest.

"In Diseases of the Nose, Throat and Ear," by A. Logan Turner, Ed., 1936, the following statement appears on page 96. "It is doubtful if a parallel condition (i.e., mucocele), has been found in the antrum." The swelling in this case was situated just above the lower margin of the orbit, and could be palpated for a certain distance into the orbit. It gave the typical sensation of a fluidcontaining cyst, and was but slightly moveable from side to side. The globe was displaced upwards. Apart from disfigurement and diplopia the patient suffered little inconvenience.

His photograph belies his mental outlook - he was not worried; he had no pain; it is unlikely for those reasons that he will trouble to come the 15 miles or so to hospital.

It is regretted that definite proof that this is a mucocele of the antrum has not, so far, been obtained in this case.

\title{
RETRO-BULBAR NEURITIS (Five Cases) due to Para-nasal Sinusitis
}

\author{
BY \\ ROSA FORD \\ LONDON
}

WE see many eyes that are quite normal, with poor, sometimes extremely defective, sight. If this blindness is in only one eye, or in both with no hemianopia, the lesion must lie in one or both optic nerves, and the condition is called retro-bulbar neuritis. 
That is sometimes the extent of our knowledge, and it is often necessary to tell the patient, "Your trouble is behind the eye, but we do not know what it is."

The following reports of five cases are made for the light they throw on their aetiology, and as some contribution to a very controversial and so far unsolved problem :-

CASE 1.-A man, aged 31 years, was seen in February, 1926, for defective vision in the right eye, of five months' duration. Vision was $2 / 60$, with nothing in the eye to account for the defect. The sinuses were reported normal both clinically and radiologically, and no other possible/ cause was discovered. He remained under observation for fifteen months, during which time vision increased to $6 / 18$

He did not attend again for two years and four months, when he stated that his vision had remained exactly as on his last visit till nine months ago, when it had recovered as unaccountably as the defect had begun, and had given him no trouble since. The defect had thus lasted for over three years. . Vision had become 6/9. He was not then seeking advice for his eyes but for catarrh, which was so troublesome it was disturbing his sleep. He had had post-nasal catarrh for six years, but this exacerbation had only begun nine months ago.

Recovery of sight had thus synchronised with the onset of severe post-nasal catarrh, suggesting that this spontaneous drainage of the sinuses had relieved the pressure on the optic nerve responsible for the blindness.

While the sinusitis had remained latent, i.e., with secretion retained, retro-bulbar neuritis had persisted; when it became obvious through release of secretion into the nose the neuritis cleared.

CASE 2.-A woman, 1 aged 31 years, ${ }^{r}$ with defective vision for the last two months. Vision was $6 / 18$ in each eye, and the fundi and media were normal. The cause was obscure. Five months later, with vision still $6 / 18$, an exploratory syringe withdrew pus from the left sphenoidal sinus and cloudy mucus from the right. There was nothing at all obvious about this sinusitis, for there had been no catarrh, and two surgeons had declared the nose particularly normal, both in bony formation and appearance of the mucous membrane. When it became obvious, after operation and treatment, the retro-bulbar neuritis cleared and vision finally became $6 / 6$ in each eye.

CASE 3.-A woman, 2 aged 39 years, with defective vision in both eyes. Right vision had never been above $6 / 12$ for three years, and left vision had been $<6 / 60$ for the last six months. She had been under expert ophthalmic, rhinological and neurological advice for seven years, but the cause had entirely escaped detection because the nose gave no sign of. sinus infection either clinically, radiologically or even to proof-puncture, and there had been no catarrh.

With intra-nasal medication free catarrh developed, and vision rose in seven weeks to $6 / 6$ in the right eye and $6 / 24$ in the left; the remaining defect being due to a patch of choroidal atrophy which involved the macula.

CASE 4.-A woman, ${ }^{3}$ aged 31 years, whase right vision had been defective since two years old. She could count fingers at one foot, but only in the outer part of the field. With fundus and media normal and some convergent strabismus, the eye had been deemed "lazy," and none knew why.

The field showed a large and intense upper scotoma, wedge-shaped, extending from the periphery to its apex just below the fixation point, thus cutting off central vision.

There had been nothing to suggest any implication of the sinuses in the amblyopia, for there had never been any catarrh, and there was no definite radiological evidence of sinusitis.

With intra-nasal medication free muco-purulent catarrh developed, and an indication of returning sight came when the patient could see her right arm swinging as she walked, for the first time in her life.

In eight months the scotoma had become progressively narrower and less intense, so that while at first no hand movements could be seen in its area, a $20 \mathrm{~mm}$. white object later became visible, and then a ten and a six, except for a narrow central core extending to only $11 \mathrm{deg}$. above the fixation point:

In 14 months the scotoma still existed, but only for a $3 \mathrm{~mm}$. white object, 
and this just crossed the fixation point, so that the patient saw an object best by looking slightly above it. In this way she was finally able to read a few words of J.I, and vision was $5 / 60$.

The slowly progressive lightening of the scotoma, as the result of continuous drainage of the sinuses, indicated the very gradual subsidence of the oedema surrounding the inflamed sinuses.

CASE 5.-A woman, aged 62 years, who had suddenly become blind in the left eye two years before. Central vision was $<6 / 60$, and there was a large scotoma extending from the lower periphery to $20 \mathrm{deg}$. above the fixation point.

The only clues to sinus disease as the cause were:-

(1) Markedly contraçted fields.

(2) Evidence of toxaemia in her insomnia, fatigue, headache, depression and irritability.

(3) Evidence of marked congestion at the back of the eyes, causing such severe photophobia that she only went out at night.

With intra-nasal medication free post-nasal catarrh developed, and within three months all her toxic symptoms and the photophobia had vanished, and the scotoma had shrunk to a small central area extending no further than $10 \mathrm{deg}$. from the centre.

Retro-bulbar neuritis often recovers spontaneously, and this has frequently led to the adoption of a waiting policy. That this is dangerous "wishful thinking" is shown by this patient's two years of blindness and ill-health, and the fact that, though health has been restored, there is still a small central scotoma eight years after her first attendance.

The entire absence of catarrh had misled the two ophthalmic surgeons previously consulted, who had thus been unable to give her any help.

To these cases may be added the description given by Nettleship 4 of an analogous condition in a Jersey heifer. The animal had suddenly become blind and ill without any ascertainable reason. On the third day a profuse muco-purulent discharge from the nostrils occurred and was followed by gradually restored sight and health.

A case reported by Sandford ${ }^{5}$ shows to what an extreme degree sinus disease may proceed and yet give no evidence of its existence except by blindness: The man died, and at post-mortem a broken-down sphenoidal sinus was found, from which a subdural abscess had spread across the middle fossa and was pointing . externally in the temple. There had been nothing to indicate this during life but optic neuritis in both eyes for the last two and a half years.

\section{Comments}

The five cases now reported, showing recovered vision after defects lasting in some as long as 2,3 , or even 29 years, point less to a neuritis than to pressure on optic nerve fibres analogous to that exerted by a pituitary tumour, removal of which even after prolonged pressure on the optic nerve is followed by restored sight.

The optic canal through which the nerve reaches the orbit is in close relation to the sphenoidal and/or the posterior ethmoidal sinuses. 'It is within the bony margins of this canal that the nerve can be subjected to pressure. If this pressure were due 
to septic exudation from the sinuses there could hardly fail to be an extension of inflammation to the nerve itself, in spite of the protection of its dural sheath, during these long periods of close contact, i.e., an actual neuritis. Visual improvement in these cases, however, was so prompt and progressed so steadily as drainage continued that a gradual subsidence of chronic oedema

1 surrounding the sinuses and compressing the nerve in its canal is the more likely explanation.

This steadily progressive return of sight is graphically demonstrated in the field charts ${ }^{3}$ of Case 4 , in which the large and intense scotoma is seen gradually to dwindle both in size and intensity during a period of 14 months' continuous drainage.

The term "retro-bulbar neuritis" is thus scarcely a correct description of the condition, and might more accurately be replaced by the term "pressure paresis of the optic nerve."

There are many degrees of this paresis. In these five cases it was severe, but slighter pressure is common, and many eyes are called " lazy" because they fail to see $6 / 6$ with no apparent cause for the defect. In most such cases the amblyopia will be found to disappear when męans are taken to secure adequate drainage of the sinuses.

Retro-bulbar neuritis is said to be very rarely the result of sinus disease, and then only when the latter is obvious. .

These cases show that, on the contrary, it is when the sinusitis is hidden, i.e., when secretion is retained, that retro-bulbar neuritis develops; as soon as it becomes obvious, either spontaneously or by nasal drainage, the' retro-bulbar neuritis terfds to disappear.

It was this peculiar characteristic of sinus disease, to remain " silent" for prolonged periods, even when infection was active, that accounted for the previous failure to arrive at the correct diagnosis in all five cases.

"Silent" sinusitis has remained unmasked for many decades because methods of diagnosis and treatment have been inadequate.

Though diagnosis cannot be made by clinical examination when there is no catarrh, and often even radiology and proof-puncture are unhelpful, it can be made, as it was in most. of these cases, by the fields of vision, aided by a careful study of the history and past history; even without the help of the more inconvenient methods of culture of sinus washings after the method of Watson Williams, or a lipiodol X-ray.

Treatment by resection of the septum, and even opening of the sinuses, may give little or no confirmation of the diagnosis. A freed air passage does not necessarily free the sinuses. One infected sinus missed may queer the result, and even if opened may close again. Nothing is adequate but the effective continuous 
unloading of the sinuses, which occurred spontaneously in one case, and was obtained in the others by appropriate intra-nasal treatment.

The gradual widening of the fields as the sinuses are drained is a source of satisfaction and encouragement to the patient, who finds in it a visual record of his progress, and the gradual disappearance of his toxic symptoms makes him the more willing to persevere.

As the sinusitis in all these cases escaped detection for long periods, in some even for years, and only became evident through the unloading of its secretion, it is not unreasonable to surmise that the same may be true of many another unexplained case of retrobulbar neuritis. The large percentage of obscure cases may thus be substantially reduced, if not altogether eliminated.

\title{
REFERENCES
}

1. Brit. Jl. Ophthal., p. 56, February, 1942.

2. Proc. Roy. Soc. Med., May, 1931.

3. Trans. Ophthal. Soc. U.K., Vol.. LIII, 1933.

4. NetTleship.-Ophthal. Hosp. Rpts., p. 22, 1913.

4. SANDFORD.-Trans. Ophthal. Soc. U.K., p. 119, 1894.

\section{RECONSTRUCTION OF THE LOWER LID BY HUGHES' METHOD* ।}

\author{
BY \\ JOHN FOSTER \\ LEEDS
}

LOCALLY malignant neoplasms of the lower lid, unless thoroughly treated in the early stages, may start a train of consequences ending in evisceration of the orbit. Such cases, though rare, are still occasionally seen.

. In a day when operations on the lacrymal sac are delegated to rhinologists, those on the lids to plastic surgeons, and those in the orbit to the neuro-surgical fraternity, it is a pleasure to pay tribute to an operation on the ocular adnexa, which not only deals effectively with such a serious condition, but is both purely ophthalmic, and relatively simple.

It is relatively easy in principle if the lower lid is destroyed or removed, to find enough skin to restore the outer layer from the cheek, and enough bulbar conjunctiva to restore the inner layer from the eye. The intermediate layer of lashes and tarsal cartilage, however, are more difficult to replace. Hughes' procedure (an

\footnotetext{
* Received for publication, May 3, 1944.
} 